\title{
ODD (HALL) VISCOSITY
}

Authors: A.Nicolis \& Dam Thanh Son, arXiv:cond-mat/1103.2137

\section{Recommended with a Commentary by David Khmelnitskii, Trinity College, Cambridge}

Attentive reader of Cond-Mat Preprint Archive on the Net must register an increasing number of theoretical papers which have the words "Odd (Hall) Viscosity" in their headlines. The point is that the stress tensor $\Pi_{i j}$ of a neutral viscous liquid includes the pressure, Bernoulli and viscous contributions

$$
\Pi_{i j}=p \delta_{i j}+\rho \dot{u}_{i} \dot{u}_{j}+\sigma_{i j}, \quad \sigma_{i j}=\eta_{i j k l} \dot{u}_{k l}
$$

where the notation $u_{i j}$ denotes the strain $u_{i j}=\left(\partial_{i} u_{j}+\partial_{j} u_{i}\right) / 2$. The tensor of viscosity coefficients $\hat{\eta}$ is symmetric with respect to permutations $i \leftrightarrow j, k \leftrightarrow$ $l$. As for permutation $i j \leftrightarrow k l$, the relevant symmetry is determined by the Onsager reciprocal relation. If the time inversion is preserved, the viscosity $\hat{\eta}$ is symmetric with respect to permutation $i j \leftrightarrow k l$, and an isotropic liquid is characterised by two coefficients of viscosity $\eta$ and $\zeta$. In case, when the time inversion symmetry is broken (by external magnetic field, spontaneous magnetisation, rotation, etc) the tensor $\hat{\eta}$ has an asymmetric part and the new dissipationless viscosity coefficient $\eta^{A}$ appears. All this is known and even mentioned in the textbooks and monographs. New wave of interest to odd viscosity arose in connection with the Quantum Hall Physics [1], when the dissipative viscosities $\eta$ and $\zeta$ vanish, while the odd viscosity $\eta^{A}$ remain finite and even quantised.

Anybody, who wants to obtain an introduction into this subject, must read first an excellent pedagogic article by Jossi Avron [2]. He considers an isotropic non-conducting liquid with a breaking of the time inversion symmetry, when $\eta^{A} \neq 0$. If $\eta$ and $\zeta$ vanish, this leads to appearance of the new branch of collective motion: viscous waves. Unfortunately, there is no viscous waves in an incompressible fluids.

In an earlier paper [1], its authors considered a 2D gas of non-interacting electrons in perpendicular magnetic field and its response to adiabatic varia- 
tion of the parameters under condition when the lowest Landau level (LLL) is totally filled and, therefore, the energy levels have a gap. Analogously to the proof of the Hall conductance quantisation, these authors have found out that the Hall viscosity corresponds to the Berry curvature and, therefore, it is quantised:

$$
\eta^{A}=\frac{\pi \rho \hbar s}{4}
$$

where $\rho$ is the density, i.e. the number of electrons per unit area and $s=1$ for the LLL.

New stage of interest to the subject begun with the paper by Nick Read [3], who considered adiabatic transport and Hall viscosity in the Fractional Quantum Hall (FQHE) states and $p_{x}+i p_{y}$ paired superfluids. He concludes that the mutual interaction of electrons and even absence of the gap in the energy spectrum do not prevent the Hall viscosity from quantisation and the parameter $s$ in Eq (1) coincides with the "conformal spin density". In the Laughlin state, the conformal spin density is equal to inverse filling factor $\left(s=\nu^{-1}=e B / 2 \pi \rho c \hbar\right)$. Read considers then more general trial functions for FQHE, which include conformal blocks characterised by the scaling dimension $h_{\psi}\left(h_{\psi}=1 / 2\right.$ for Majorana fermions). He concludes that for these states $s=\nu^{-1}+2 h_{\psi}$.

Publication of the Read's article was followed up by the large number of papers $[4,5,6,7, ?]$. The last one highlighted above brings into consideration an effective quantum field theory which exhibits a nonvanishing Hall viscosity.

The literature still does not report about any experimental measurements of the Hall viscosity for 2D electrons. On the theory side, we still have many questions. The most striking of them is about efficient experimental setup which would allow to measure this quantity.

\section{References}

[1] J.E.Avron, R.Seiler \& P.G.Zograf, arXiv:cond-mat/950211

[2] J.E. Avron, arXiv:physics/971250v2

[3] N. Read, arXiv:cond-mat/0805.2507

[4] F.D.M.Haldane, arXiv:cond-mat/0906.1854 
[5] T.Kimura, arXiv:cond-mat/1008.3454

[6] N. Read \& E.H.Rezayi, arXiv:cond-mat/1008.0210

[7] M.V.Milovanovic, arXiv:cond-mat/1008:3454 